\title{
CRYSTAL-BASED COLLIMATION IN MODERN HADRON COLLIDERS
}

\author{
W. Scandale, CERN, Geneva, Switzerland
}

\begin{abstract}
The use of bent crystals for beam manipulations in particle accelerators or in external beam-lines is an old concept, still under scrutiny. In the last three decades, the knowledge on the features of crystal-particle interaction has been continuously growing on the basis of a large number of experimental findings. In all cases, the results of measurements have been found in agreement and, sometimes, have been anticipated by numerical models and simulations. Bent crystals can impart angular deviations to the incoming particles, through channelling or volume reflection mechanisms. The efficiency of the latter mechanism has been found to be intrinsically large, whilst the channelling efficiency has been improved by the increased technological expertise in crystal cutting and bending. In this paper we review the recent milestones of the worldwide effort to propose a routine use of bent crystal in particle accelerators, with a specific attention to the proposals of promoting the use of bent crystals as primary element in a collimation system.
\end{abstract}

\section{HISTORICAL PERSPECTIVE}

In the early Eighties, a new generation of synchrotrons with superconducting magnets, providing high luminosity and high-energy hadron collisions well beyond the $\mathrm{TeV}$ in the center-of-mass, was considered as the forefront of the particle physics. The construction of infrastructures of this type was proposed in the USA (SSC) [1], in URSS (UNK) [2] and in Europe (CERN-LHC) [3]. In all of them, already at the early stage of the conceptual design, two areas were identified, in wich the use of bend crystals would have been beneficial if not essential: the beam extraction to feed external beam lines and the beam collimation to collect the halo particles surrounding the beam core, respectively. This fact triggered, in the early Ninties, a whelm of investigations aiming at the characterization and the optimization of the crystal parameters for their optimal use in circular accelerators.

Exploring CP-violation with high-energy external primary beams interacting on a fixed target in an underground experimental area was highly rated by the physics community and considered as a very promising application in LHC [4]. The main issue was to find an efficient way of extracting the circulating particles in parasitic mode during the beam-beam collision runs [5]. Experimental checks were organized in both side of the Atlantic to investigate if bent crystals could provide the required flux of extracted particles. In 1990, the experiment RD22 was launched in the CERN-SPS, with the aim of reaching the unprecedented range of extraction efficiency, above $10 \%$ of the circulating current, exceeding by two order of magnitudes the state-of-the-art of the time [6]. Just after the first positive results of $\mathrm{RD} 22$, in 1992, another experiment, named E853, was approved at FNAL with the goal of testing a luminosity driven extraction mechanism compatible with the cryogenic environment of the Tevatron eventually providing a minimal impact on the collision physics runs.

Both the experiments were very successful. In RD22 the required efficiency of $10 \%$ was indeed observed for the first time and routinely reproduced, whilst the multiturn effect, providing channelling of protons even after several turn-by-turn crystal traversal, was demonstrated in a clear manner [7]. At a given beam energy, the channelling efficiency was found to depend on the interplay of the multiple scattering in the crystal traversal with the dechannelling probability [7]. The channelling extraction was also observed with fully stripper $\mathrm{Pb}$-ion beams, although associated to a rather large break-up cross-section [8]. In E853 the first ever extraction driven by beam-beam process was obtained and the highestenergy proton channelling effect ever produced $[9,10]$. The use of crystals to assist the collimation process, suggested in the late Eighties by several Authors, see for instance Ref [11], was preliminarily tested in the Tevatron during the E853 runs with encouraging results.

These developments were suspended in the second half of the Nineties for various reasons related to the economical context and to new emerging priorities in high-energy physics. Only a moderate activity persisted in Russia at IHEP where crystal tests continued in the U70 synchrotron and at PNPI where new types of crystals were built and characterized. However, the accumulated experience had the large merit of demonstrating that the crystal channelling mechanism is compatible with the operation of modern hadron colliders and could be successfully applied for some specific and very demanding beam manipulation.

At the end of the Nineties, crystal investigation was vigorously resumed. First, a crystal collimation scheme was implemented and tested at LBNL with the aim of reducing the background in the RHIC detectors. Later, the experiment continued at FNAL in the Tevatron, with the same crystal and goniometer. At the same time, fundamental studies on high-energy particle interaction with crystals were resurrected also in Europe. In 2000, a scientific collaboration involving CERN, INFN, IHEP, PNPI and JINR, was created to handle three specific INTAS-CERN programmes, co-funded by EU and CERN, and later by the Russian academy of Science and the NTA-INFN programme. In 2006, this activity evolved in a large campaign of experiments in the North Area of the SPS and, with the support of CARE-HHH, the international Collaboration H8RD22 was created to handle it. The worldwide revival of interest in crystals of 
the last years sustained by the strong networking support of CARE-HHH was fruitful. In September 2008, the CERN Research Board approved a crystal collimation experiment in the SPS named UA9 [12]. To handle it, the UA9 Collaboration was created from the extension of H8RD22 to LBNL, FNAL and SLAC with the support of LARP. Slightly later, the FNAL management endorsed the activity on crystals inherited from LBNL and approved the T980 experiment to test crystal collimation in the Tevatron, complementing the UA9 programme.

UA9 and T980 have the common goal of investigating improved collimation concepts for the LHC. They have complementary approaches to face the numerous unknown issues still to be elucidated.

\section{OPTIMAL CRYSTALS FOR HIGH- ENERGY PARTICLE MANIPULATIONS}

The crystals used in RD22 and E853 experiments were at the forefront of the technology in the late Eighties. They were dislocation-free crystals, precisely cut with the faces parallel to (110) planes, within a hundreds of $\mu \mathrm{rad}$, with the beam face properly etched and optically polished and a rather flattish horizontal-vertical aspect ratio of the shape to favour mechanical bending. The crystal length was of a few cm (3 in RD22 and 4 in E853). The bending angle was of a few hundreds of $\mu \mathrm{rad}$ (200 in RD22 and 640 in E853) and was imparted by a multi-point mechanical bender, as in Figure 1. The side effect of the multi-point clamping of the crystal to the bender was the local increase of the crystal curvature and hence the overall reduction of the channelling efficiency.

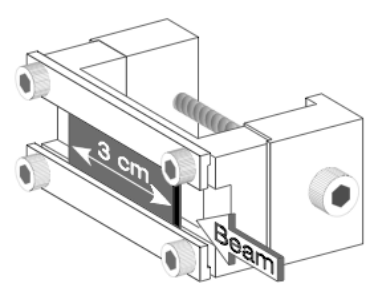

Figure 1: crystal with multi-point clamping used in RD22.

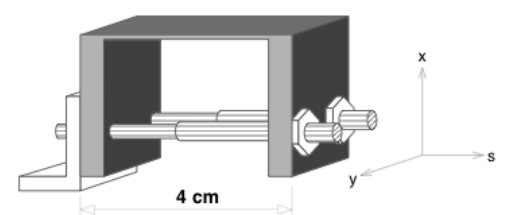

Figure 2: U-shaped crystal used in RD22.
As a potential remedy, U-shaped crystals without mechanical clamps in the beam face, as in Figure 2, were suggested by the ESRF in Grenoble and tested in RD22, without showing measurable improvements of the channelling efficiency [13]. In fact, O-shaped crystals proposed by PNPI were an intrinsically better solution for a regular curvature of the crystal. Although available too late for RD22 and T853, the PNPI O-shaped crystal shown in Figure 3 was used in RHIC and later in T980.
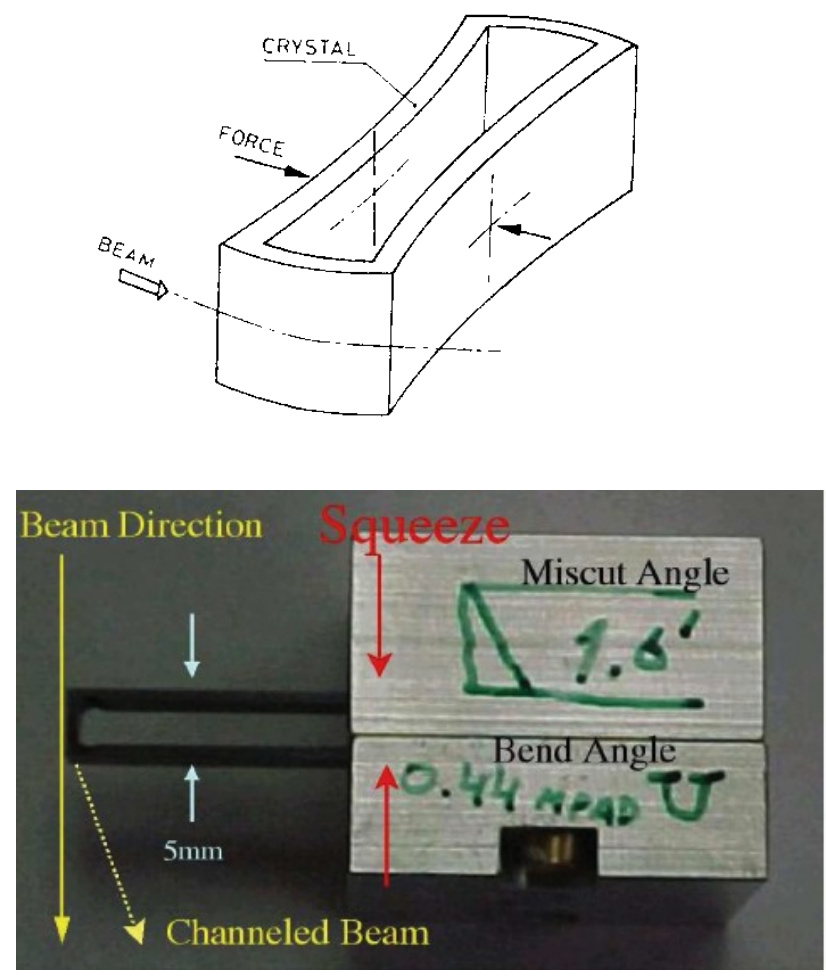

Figure 3: O-shaped crystal produced in PNPI and used in RHIC and later transferred in the Tevatron for the T980 test. Top part: the O-shaped bending principle. Bottom part: the RHIC/T980 crystal in its bender.

The mentioned INTAS-CERN programmes played a crucial role in fostering the European cooperation and in supporting systematic investigations and new proposals to develop and test optimal crystals for high-energy particle manipulations. The main issue addressed was related to the possible use of short crystals for a much larger channelling efficiency at the highest beam energy [14,15]. Crucial achievements have been obtained in that frame. Strip crystal plates of 1 to a few mm thickness have been cut and bent as to produce an homogeneous anticlastic curvature of the order of or larger than a few hundred $\mu \mathrm{rad}[16,17]$. One of the first strip crystals in its original holder used in U70 at IHEP is shown in Figure 4. Shorter crystals of a few tenths of mm length have also been cut with the external faces parallel to the atomic lattice and bent by hundreds of $\mu \mathrm{rad}$ through the quasi-mosaic elastic effect [18]. A couple of crystals of this type produced at PNPI and used in the North Area of the SPS are shown in Figure 5. Finally a procedure for a controlled chemical etching of the crystal surface has been identified and 
optimized to eliminate the amorphous layers from the crystal edges, produced during the crystal cut [16]. An example of the crystal quality at the surface is shown in Figure 6, for a crystal etched in Ferrara University.

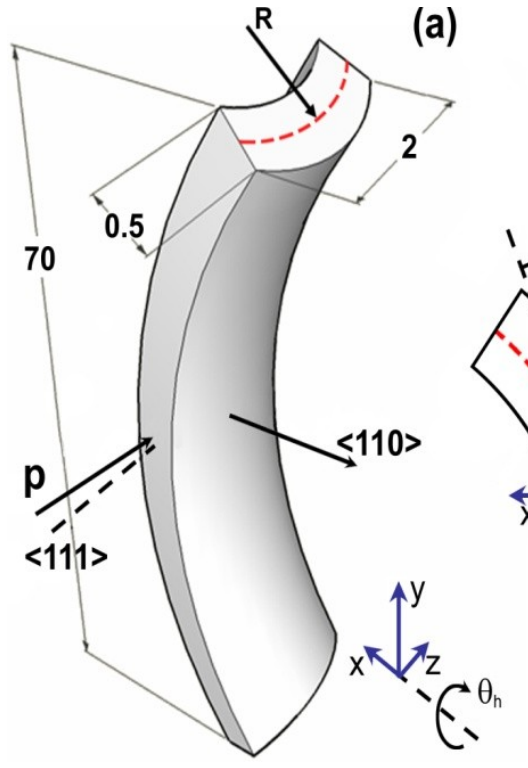

(b)

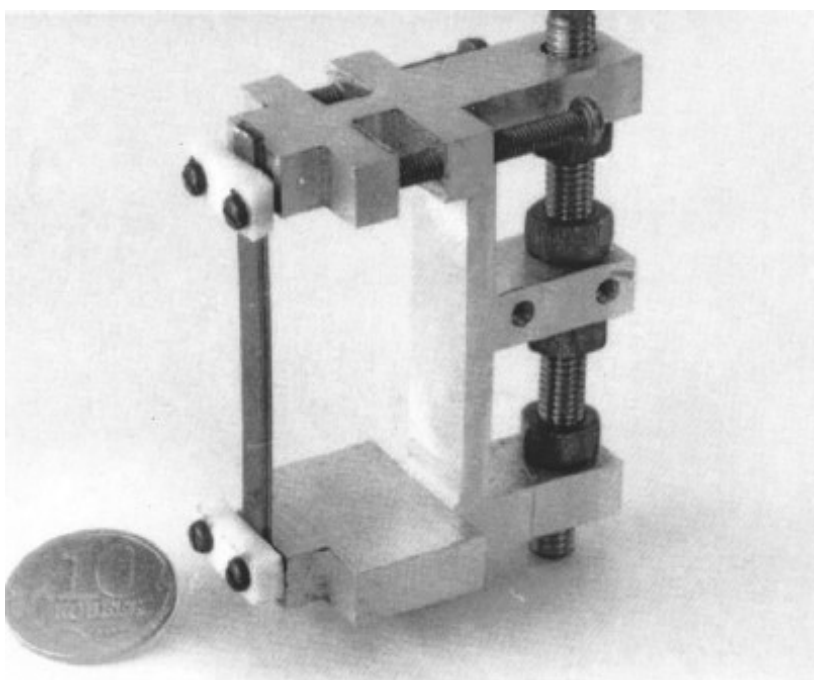

Figure 4: Strip crystal in its holder. Top part: the bending principle: the main radius of curvature along the strip axis, impart an anticlastic radius of curvature to the face exposed to the beam. Bottom part the IHEP strip crystal in its original bender.

All these steps represent indeed an ensemble of technological breakthrough for the optimal use of crystals in particle accelerators. Short crystals of a few $\mathrm{mm}$ or a few fractions of $\mathrm{mm}$ length allow extending the range of energy, in which crystals have large channelling efficiency [14]. The constant curvature obtained through the elastic property of the crystal itself avoids local perturbations of the bending radius eventually introduced whenever external supports are used to impart the flexural stress on the face exposed to beams, thereby reducing the dechannelling probability. The removal of the amorphous layer at the crystal surface implies the possibility of channelling as soon as the particles hit the crystal surface, which is a mandatory request when particles intersect the crystal at a small greezing angle.
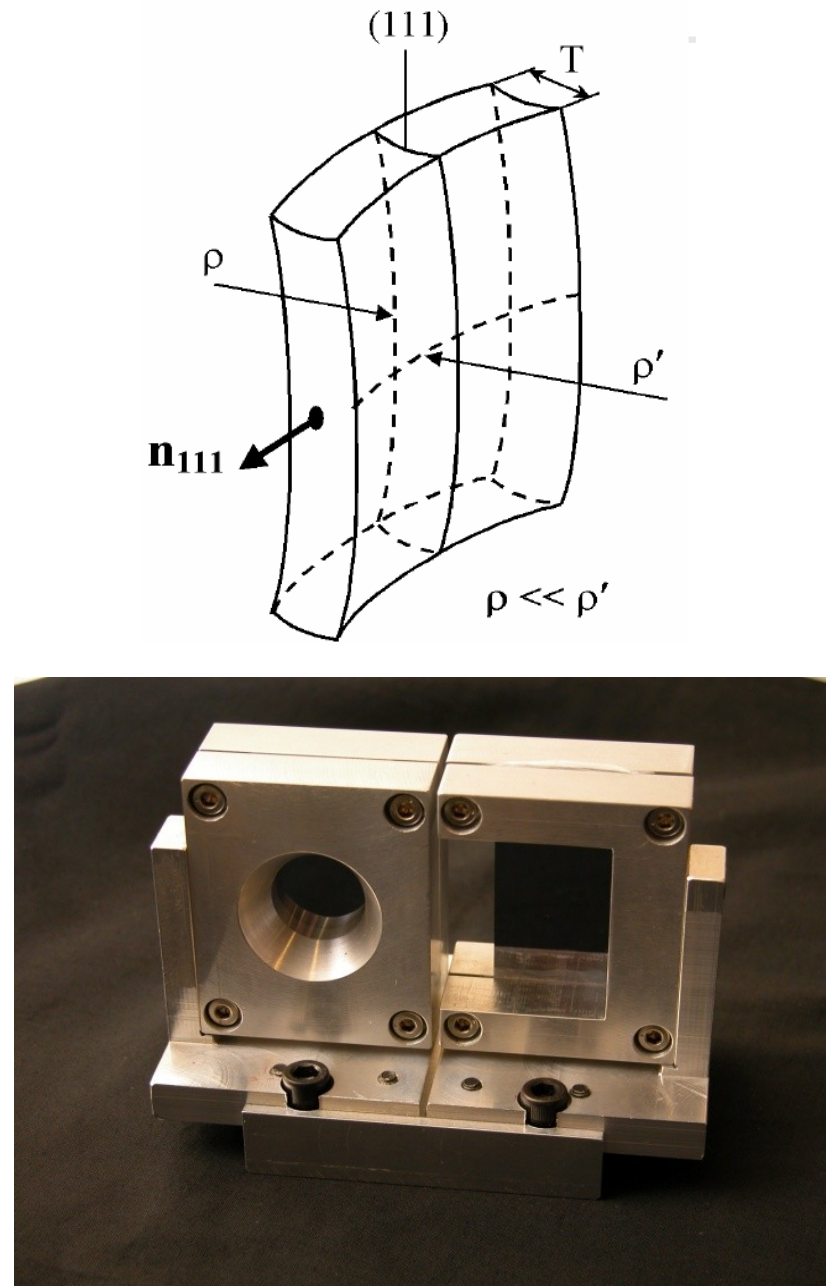

Figure 5: Silicon crystals with elastic quasimosaic bending. Top part: the bending principle: the principal curvature imparted by the support to the crystal axis imposes the anticlastic curvature of the larger face and at the same time the quasimosaic curvature of the (111) planes parallel to the thinner face due to the anisotropy of the elastic tensor force. Bottom part: a pair of crystals in their bender used in North Area of the SPS.

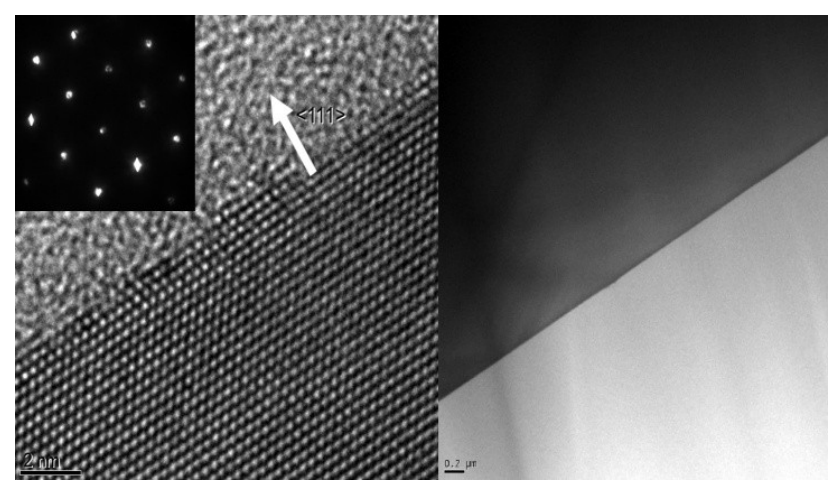

Figure 6: Etched crystal produced in Ferrara. Right side: the edge of the crystal. Left side: the AFM blow-up. 
Short crystals, chemically polished, with anticlastic bending of quasimosaic elastic curvature were produced and extensively tested in the U70 accelerator at IHEP with $70 \mathrm{GeV}$ protons and in the extracted beam lines in the North Area of the SPS with $400 \mathrm{GeV}$ protons demonstrating a channelling efficiency as large as $85 \%$ $[14,19]$.

\section{CRYSTAL TESTS IN THE NORTH AREA}

In 2006, the CERN management approved an experimental campaign with bent crystals in the North Area of the SPS. An international collaboration called H8RD22, including CERN, INFN, PNPI, IHEP and JINP, which received financial support also from CARE-HHH, INTAS-CERN and INFN-NTA, endorsed this activity, which is still in progress and already provided a terrific whelm of information on high-energy proton interactions with bent crystals. The initial detector, based on silicon strips and scintillator counters, is pictorially shown in Figure 7 [20]. Single particle trajectories were identified in the two detector arms, by which the incoming and outgoing angles were reconstructed. One of the most striking results was obtained with a $9 \mathrm{~mm}$ long quasimosaic crystal bent by $150 \mu \mathrm{rad}$, deflecting $400 \mathrm{GeV}$ proton beam extracted from the SPS. The measured deflection angle induced by a bent crystal is shown in Figure 8 as a function of the crystal-beam orientation. The colour code identifies the fraction of deflected particles. Six different interaction regimes are visible, of which the area 2 is due to channelling and the area 4 is due to volume reflection [21]. The measured efficiency, intended as the fraction of deflected particles, were about $50 \%$ for channelling and more than $97 \%$ for reflection [21]. As a consequence of the latter observation, the volume reflection process has been considered as very promising for sophisticated beam manipulations.

Other important results of H8RD22 must be mentioned here. In bent crystals much shorter that the dechannelling length due to multiple scattering, the dechannelling length due to nuclear scattering could be directly measured and an unprecedentedly high deflection efficiency was detected [19]. Short crystals were also used to bring into evidence the dependence of the channelling and reflection efficiency on crystal curvature [22]. Assembly of many crystals mechanically aligned to each other were used to produce a cascade of multiple reflections and to measure the multi-process probability, by which larger deflections would be imparted to the incoming particles [23]. Finally, thanks to a multi-stage goniometer, by which the polar and the azimuthal crystal-beam angle were adjusted the axial channelling process was produced and studied in detail [24].

The H8RD22 Collaboration also spent a considerable effort to investigate how the interaction with crystals changes if the incoming particles are light leptons, of positive or negative charge or negative hadrons. These investigations are of a direct interest for future colliders such as the ILC of the Muon Collider and for the Tevatron as well where antiprotons are circulating. Some of the results are yet unpublished and hence cannot be discussed here. Quantitative data on the probability of planar channelling, volume reflection and axial channelling process will be available as soon as possible. The data referring to $180 \mathrm{GeV}$ positrons interacting with the mentioned quasimosaic crystal $9 \mathrm{~mm}$ long with $150 \mu \mathrm{rad}$ bend angle are instead available in Ref [25], where the measured efficiency of the various deflection process is discussed and the associate spectra of the emitted radiation described.

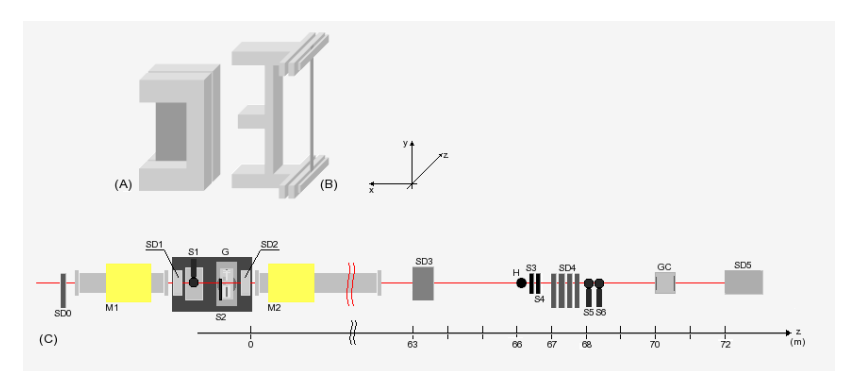

Figure 7: The detector of the H8RD22 Collaboration. A goniometer $(\mathrm{G})$ orients bent crystal along the direction of incoming beams. The detector telescope has two arms, made of silicon strips and scintillator counters, by which the individual particle trajectories are recorded, before and after the crystal hit. The measured difference in angle gives the effect of the bent. Both strip (A) and quasimosaic (B) crystals were studied.

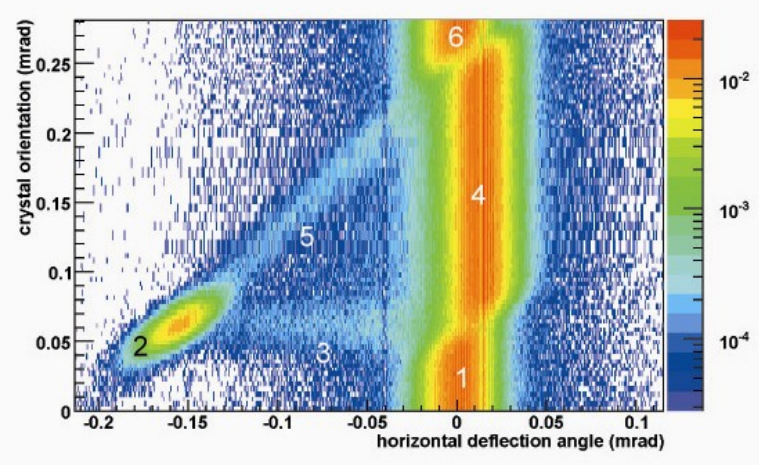

Figure 8: Data collected by the H8RD22 Collaboration. The horizontal deflection angle induced by the crystal is plotted as a function of the crystal-beam orientation with a colour code giving the fraction of deflected particles. Six areas are visible: 1 and 6 where the crystal acts as an amorphous material, 2 where there is channelling, 4 where there is volume reflection, 3 where there is dechanneling, 5 where there is volume capture.

\section{CRYSTAL COLLIMATION TESTS}

In the recent years, the attempt of using bent crystal for halo reduction was pursued in RHIC, using O-shaped crystals from PNPI. The experimental results have been 
somehow controversial: the observed collimation effect was inexistent and in certain cases worsened respect to that of an amorphous primary, although the measured channelling efficiency was consistently exceeding $30 \%$. For this reason the attempt of assisting collimation with crystals was abandoned at RHIC [25].

However, in 2005, the equipment of RHIC was dismounted and reinstalled in the Tevatron at FNAL, and, in 2006, a channelling efficiency larger than $50 \%$ was measured and a background reduction by a factor of two observed in the CDF detector, in agreement with simulations [26].

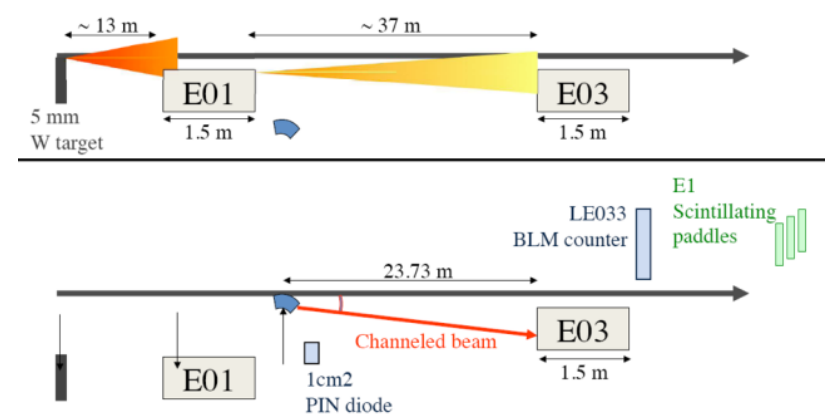

Figure 9. Layout of the Tevatron E0 region with tungsten target (top) and crystal (bottom).

Unfortunately, problems with the mechanics of the goniometer revealed in earlier studies, forced the FNAL team to stop the investigations and improve the hardware. This operation was successful and afterwards the angular positioning of the crystal cold be made with a resolution of $2.1 \mu \mathrm{rad}$. In addition, the O-shaped crystal used in 2005 was dismounted and characterized again, with the help of PNPI and INFN. Another PIN diode telescope was installed immediately downstream of the crystal, and a scintillation counter telescope and a beam loss monitor was located downstream of the secondary collimator. The new layout is show in Figure 9. In the most recent crystal collimation tests at the Tevatron channelling has been observed once more. The angle scan results are reproducible. There is the evidence that channelling improves beam loss localization. Simulations show that the miscut angle of the O-shaped crystal should be small and its orientation should be negative. The plans for future are to optimize the collimator positions and to replace the O-shaped crystal with a new one with a much smaller miscut angle and a negative orientation. Further extensions of the experiment include installing a second (vertical) goniometer with two alternating crystals: an $\mathrm{O}$ shaped crystal (to exploit channelling) and a multi-strip array (to exploit VR). With this a two-plane collimation will be possible and a reproducible loss reduction in the superconducting ring and the $\mathrm{CDF} / \mathrm{D} 0$ detectors is expected [27].

\section{UA9}

As already mentioned, UA9, approved in September 08 by the CERN Research Board, will study features of crystal channelling in the SPS using single-particle detectors and compare the effect of different crystal technologies, strip or quasimosaic, using either channelling or volume reflection or even multi-reflection with a sequence of aligned crystals.

\section{The UA9 layout}

The layout of UA9 is shown in Figure 10. The halo particles should be deflected by the crystal and kept by the secondary collimator in the horizontal plane where the SPS aperture is larger. All the components of UA9 are retractable for an easy routine operation at high intensity. The layout is as compact as possible to save on cost of cables and of other infrastructures.

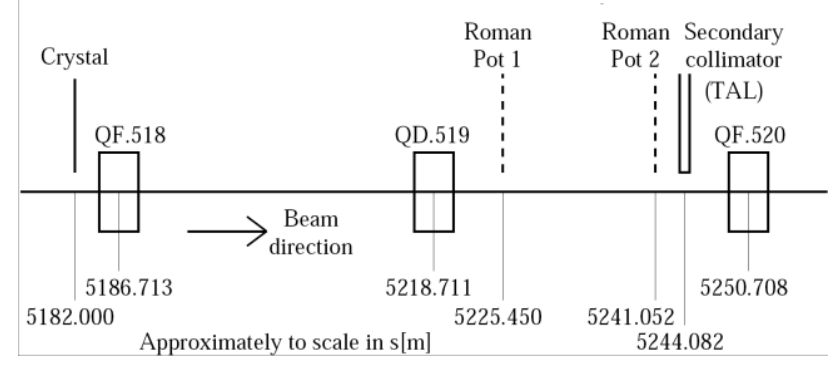

Figure 10: Layout of UA9

The core of the UA9 is installed in the two dipoleempty cells of the long straight section 5 of the SPS, just across the cavern formerly hosting UA1.

A large tank is in place, equipped with several devices. There are two goniometers each with a holder and a single-crystal, one strip and one quasi-mosaic, which will be used in alternative as crystalline collimators. The goniometer can be moved towards the beam and oriented to its direction with a resolution of a tenth of $\mu \mathrm{rad}$, i.e. of the order of the critical angle for channeling at the nominal beam energy of $120 \mathrm{GeV}$. A movable thin target in tungsten is added for comparison, which will be used as an amorphous primary collimator. The tank also contains three feed-through for 100 twisted pair cables, for future use, should active piezoelectric motors be required to mutually orient multi-crystal assemblies. In the wall side of the tank an external laser system is used to align the crystals with an autocollimator and two pentaprisms at the installation time. Later alignment checks will be made with the same system, through quartz windows located just in front of the crystals. In the upstream and downstream faces of the tank, two gas electron multiplier (GEM) pads and a pair of scintillator counters are mounted in air. Their counting rate is normally proportional to the background rate, about equal in the two face positions. When the crystal is in place, the rate of the downstream detectors should be increased by the rate of nuclear reactions in the crystal itself. The GEM pads and the scintillator counters provide similar information, for redundancy. The pads have 20×30 channels and may also give rough indications of the lateral distribution of the background. The tank can house also a bar of quartz movable laterally in vacuum, which 
once aligned to the crystal will be used as a Cerenkov detector and count the number of particles incoming into the crystal itself. This detector is to be installed in May 09 , just before the running in of UA9.

The collimator tank houses a movable block of tungsten $600 \mathrm{~mm}$ long, 30x30 $\mathrm{mm}^{2}$ wide (TAL). Attached to it and aligned to its front face, there is a bar of quartz, movable in vacuum, used a Cerenkov detector, by which the number of particles reaching the collimator is counted.

The roman pot 1 is in place, equipped with one siliconstrip detector, mounted in the inner side of the ring. The other pot, in the outer side of the ring, will be equipped soon with one pixel pad detector and for redundancy with a scintillation fibrometer.

Additional detectors are used in UA9. A set of scintillator counters, mounted in air in both sides of the vacuum pipe, are installed downstream of the first halfcell with bending dipoles. They will be used to detect offmomentum beam loss induced by the collimation process. In addition, a set of fast beam loss monitors (BLM) has been installed in area of UA9, to reveal the fast loss. Later in 2009 , another vacuum tank with two goniometers in construction at IHEP and the roman pot 2 in construction at SLAC and the associated detectors (silicon-strips, pixels and scintillating counters) will be added.

\section{Beam and crystal parameters}

UA9 will be operated at two energies, 120 and 270 $\mathrm{GeV}$. The former value is the nominal one of RD22 [2, 3], the crystal extraction experiment performed in the early nineties in the SPS, for which we have numerous reference data. The latter value of $270 \mathrm{GeV}$ is the energy of other machine experiments planned in the SPS simultaneously to UA9, a choice that may significantly speed-up the duration of the accelerator setting. The beam parameters will be those of Table 1, where the transverse RMS beam radius is computed in the middle of a focusing (defocusing) quadrupole for the horizontal (vertical) plane, whilst the tunes are the ones for the high intensity operation mode of the SPS. The beam intensity will be of a few $10^{10}$ up to $10^{12}$ particles. The RF beam structure will be either unbunched or bunched in one or a few tens of bunches. The accelerator will operate in storage mode.

The beam lifetime is determined by the SPS vacuum and should be larger than $80 \mathrm{~h}$. By applying an external noise to the damper, the beam lifetime should be reduced to a value ranging from a few tenths of minutes to a tenth of hour. A similar approach had been used successfully in RD22, with the hardware still currently operational in the SPS. The subsequent halo flux hitting the crystal should be sustainable by our detectors in the roman pots. In practice, we will produce one of the following conditions. A halo flux of a few $10^{2}$ to a few $10^{4}$ particles per turn evenly distributed along the revolution period (unbunched beam) or synchronous to the bunch structure (bunched beam), which can be investigated with the detectors in the roman pots, avoiding counting saturation. In alternative, larger fluxes up to a few $10^{5}$ particles per turn, which may saturate the monitors in the roman pots and hence should be studied using only the beam loss monitors of the SPS.

Table 1: beam parameters for UA9 operation.

\begin{tabular}{lcc}
\hline & High energy & Nominal energy \\
\hline$p[\mathrm{GeV} / \mathrm{c}]$ & 270 & 120 \\
\hline$Q_{x}$ & 26.13 & 26.13 \\
\hline$Q_{y}$ & 26.18 & 26.18 \\
\hline$Q_{s}$ & 0.0021 & 0.004 \\
\hline$\varepsilon_{\mathrm{N}}(1 \sigma)[\mu \mathrm{rad}]$ & 1.5 & 1.5 \\
\hline$\sigma_{x}, \sigma_{v}[\mathrm{~mm}]$ & 0.67 & 1 \\
\hline$\Delta p / p(1 \sigma)$ & 2 to $3 \times 10^{-4}$ & $4 \times 10^{-4}$ \\
\hline$\varepsilon_{L}[\mathrm{eVs}]$ & 0.4 & 0.4 \\
\hline$V_{R F}[\mathrm{MV}]$ & 1.5 & 1.5 \\
\hline
\end{tabular}

The optimal primary collimator is a silicon crystal oriented along the (111) planes, with a bend angle $\alpha=150$ $\mu \mathrm{rad}$ and a length along the beam $L=1 \mathrm{~mm}$. This gives a bend radius $R=6.67 \mathrm{~m}$ (the critical radius for $120 \mathrm{GeV}$ protons is $R_{c}=21.46 \mathrm{~cm}$ ). In these conditions, the particles deflected in the TAL should have large impact parameters, of about $6-8 \mathrm{~mm}$. The crystal bend radius, which produces the maximum extraction efficiency for $120 \mathrm{GeV}$ protons, is about 1-2 m, i.e. 5-10 times $R_{c}$ [7]. The crystal parameters, $\alpha$ and $L$, we selected are a sound compromise in between the optimal values for SPS and those eventually required for LHC. Both strip crystals with anticlastic curvature and bulk quasi-mosaic crystals may be built with parameters close to the above values.

In operational conditions, the crystal will be oriented in channelling mode and its edge will be at the distance of $x_{b c}=6 \sigma_{\text {beam }}$ from the closed orbit. Also the TAL and the two roman pots will be retracted from the beam centre, at a distance, which must be larger than that chosen for the crystal. This should guarantee in all circumstances that the crystal could intercept the halo particles before any other experimental device, thereby acting as the effective primary collimator. In addition, this should ensure that halo particles, which are not channeled in the first crystal passage and which are deflected by multiple scattering as if the crystal was an amorphous material, could hit the crystal again in the subsequent revolutions, thereby having additional chances of being eventually channeled. In other word, the multi-turn channeling mechanism should be fully active and can eventually produce the maximal collimation efficiency.

The simplest option is to position the edges of the two roman pots and of the TAL, in normalized coordinates, at the same distance $6 \sigma_{\text {beam }}+x_{o f}$ from the beam centre, shifted by the same offset $x_{o f}$ with respect to the crystal edge position. The size of $x_{o f}$ is chosen in such way that the multi-turn effect has a high probability of happening, see Table 2. Non-channeled $120 \mathrm{GeV}$ particles, in traversing the $1 \mathrm{~mm}$ long silicon crystal, are randomly scattered in all directions. The RMS scattering angle is $\theta_{\mathrm{ms}} \approx 10 \mu \mathrm{rad}$. By requiring that particles deflected by up to $4.25 \cdot \theta_{\mathrm{ms}}$ in the horizontal plane stay clear from TAL and roman pots, 
the probability of loosing multiple scattered particles in the first turn becomes negligible, i.e. of about $10^{-5}$.

Table 2: Beam size and offset along UA9.

\begin{tabular}{ccccccc}
\hline & Crystal & $\begin{array}{c}\text { QD } \\
\mathbf{5 1 9}\end{array}$ & RP1 & RP2 & $\begin{array}{c}\text { TA } \\
\mathbf{L}\end{array}$ & $\begin{array}{c}\text { QF } \\
\mathbf{5 2 0}\end{array}$ \\
\hline $\begin{array}{c}6 \sigma_{\text {beam }} \\
{[\mathrm{mm}]}\end{array}$ & 6.35 & 2.97 & 3.61 & 5.98 & 6.23 & 6.60 \\
\hline $\begin{array}{c}x_{o f} \\
{[\mathrm{~mm}]}\end{array}$ & 0 & 0.41 & 0.5 & 0.83 & 0.86 & 0.91 \\
\hline
\end{tabular}

\section{Experimental procedures}

The SPS is to be set in storage mode at $120 \mathrm{GeV}$, initially with a single circulating bunch of $5 \cdot 10^{10}$ protons. The main parameter, such as the closed orbit, the tune, the chromaticity, the beam profile, the RMS beam size, the lifetime and the beam loss pattern along the ring, can be recorded. Once the diffusive halo is activated though a continuous power injection in the beam damper, several actions will be performed. The various movable devices of UA9 will be aligned to each other using the BLM in the UA9 area. As a side effect this procedure should provide information of the closed orbit stability. By inserting and retracting the TAL close to its nominal position at about $6 \sigma_{\text {beam }}$ from the closed orbit, one can measure the diffusion speed and the counting rate of the protons that cross the UA9 detectors. This method should allow setting up the optimal loss rate and diffusion speed to avoid saturation of the UA9 counters and, at the same time, it will provide the expected value of the impact parameter and impact angle onto the crystal, when located in its nominal position.

With the UA9 equipments in their nominal transverse position, one can start orienting the crystal and searching for the optimal angle for channelling. This angle should correspond to a minimal counting rate in the counters located in the downstream tank face, once the background detected in the upstream face is subtracted. As a side effect, this procedure should be able to provide the optimal alignment of the crystal for volume reflection that is the crystal orientation in the middle of the angular range for reflection mode.

With the crystal in channelling orientation and the TAL in its nominal position, it should be possible to take a snapshot of the beam losses around the ring and in the UA9 area in order to compare them with those produced when the amorphous tungsten primary in the UA9 tank is replacing the crystal. This should provide the first set of relevant observables, by which the localization of the beam loss of a crystal collimation system could be evaluated. The evaluation can be made as well in volume reflection mode.

With the crystal in channelling orientation and the collimation in place, there are two methods to evaluate the collimation efficiency. The first method is indirect, based on the assumption that the crystal is the only object in the ring, which can absorb the halo and hence determine the beam lifetime. In a given interval of time, the number of particles touching the crystal can then be given through the lifetime record, whilst the quartz Cerenkov counter counts the flux lost in the TAL. A more direct measurement should be based on the use of the second Cerenkov close to the crystal, by which the particles touching the crystal can be given. To avoid systematic errors, the effect of multi-turn should be properly accounted for.

By inserting the roman pot in its nominal position the profile of the extracted beam on its path to the TAL can be recorded.

Before the end 2009, UA9 will be able to run and collect relevant data on crystal collimation, thanks to the heavy commitment of the full UA9 Collaboration and to the generous support of the funding agencies.

\section{ACKNOWLEDGEMENTS}

We acknowledge the support of the European Community-Research Infrastructure Activity under the FP6 "Structuring the European Research Area" programme (CARE, contract number RII3-CT-2003506395).

\section{REFERENCES}

[1] "The SSC design report", SSCDR SSCL-SR - SSC Lab, July, 1990.

[2] V. A. Yarba, "The status and development of the UNK project", Proc. of PAC 1991, vol.5, pp 291317, S. Francisco, Ca., USA, 1991.

[3] "LHC Design Report", Vol.1, "The LHC Main Ring", CERN-2004-003.

[4] T. Nakada, "Review of CP violation studies with Bmesons at LHC", Plenary talk given at 1994 International Workshop on B Physics Physics Beyond the Standard Model at the B Factory, Nagoya, October 1994.

[5] The RD22 collaboration, "A proposal to test beam extraction by crystal channeling at the SPS: a first step towards a LHC extracted beam", CERN/DRDC 91-25, Geneva, 1991.

[6] The RD22 collaboration, Physics Letters B 313 (1993) 491-497, 1993.

[7] The RD22 collaboration, Physics Letters B 357 (1995) 671-677, 1995.

[8] G. Arduini et al., Phys Rev. Lett. 79, 21, pp 4182-85, 1997.

[9] R.A. Carrigan et al. Nucl. Instrum. Methods Phys. Res. B 90, 128 (1994).

[10]R.A. Carrigan et al., Phys. Rev. ST, volume 1, 022801 (1998).

[11] M.A. Maslov, N.V. Mokhov, I.A. Yazynin, SSCL484 (1991).

[12] W. Scandale and M. Prest, "Proposal of the Crystal Experiment”, CERN-SPSC-2008-014/SPSC-P-335 (2008).

[13] J. T. Klem, "Proton extraction from a high-energy beam with bent crystals", CERN-Thesis-98-018 (1998). 
[14]A.G. Afonin et al. Phys. Rev. Lett. 87, 094802 (2001).

[15] Y.M. Ivanov et al., Phys. Rev. Lett. 97, 144801 (2006).

[16] S. Baricordi et al., Appl. Phys. Lett. 91, 061908 (2007).

[17] A. G. Afonin et al., JETP Lett. 67, 781 (1998).

[18]Y. M. Ivanov, A.A. Petrunin, and V.V. Skorobogatov, JETP Lett. 81, 99 (2005).

[19] W. Scandale et al. "Measurement of deflection efficiency and nuclear dechanneling length for high energy protons using short crystals", submit. to PRL (2009).

[20] W. Scandale et al., RSI 79, 023303 (2008).
[21] W. Scandale et al., PRL 98, 154801 (2007).

[22] W. Scandale et al., PRL 101, 234801 (2008).

[23] W. Scandale et al., PRL 102, 084801 (2009).

[24] W. Scandale et al., PRL 101, 164801 (2008).

[25]R. P. Fliller III et al., NIM B, 234, 1-2 pp 47-56, (2005).

[26] R.A. Carrigan, A.I. Drozhdin, R.P. Fliller, N.V. Mokhov, V.D. Shiltsev, D.A. Still, Fermilab-Conf309-AD (2006); Int. Conf. on Charged and Neutral Particle Channeling Phenomena, "Channeling 2006", Proc. of SPIE, 663401-1 (2007).

[27] N. V. Mokhov, et al, "Crystal collimation studies at the Tevatron (T-980)", subm. to PAC09, (2009). 